Research Paper

\title{
Expression of VEGFR-3 in intrahepatic cholangiocarcinoma correlates with unfavorable prognosis through lymphangiogenesis
}

Meng Sha*, Seogsong Jeong*, Xiao-song Chen*, Ying Tong, Jie Cao, Han-yong Sun, Lei Xia, Ning Xu, Xin Wang, Long-zhi Han, Zhi-feng Xi, Jian-jun Zhang, Xiao-ni Kong ${ }^{\bowtie}$, Qiang Xia ${ }^{\bowtie}$

Department of Liver Surgery, Ren Ji Hospital, School of Medicine, Shanghai Jiao Tong University, 1630 Dongfang Road, Shanghai, 200127, China

*Equal contributors: Meng Sha, Seosong Jeong, Xiao-song Chen

$\triangle$ Corresponding authors: Xiao-ni Kong and Qiang Xia, Department of Liver Surgery, Ren Ji Hospital, School of Medicine, Shanghai Jiao Tong University, 1630 Dongfang Road, Shanghai, 200127, China. Tel.: +862168383775; Fax: +862158737232; E-mail address: xiaqiang@shsmu.edu.cn

(C) Ivyspring International Publisher. This is an open access article distributed under the terms of the Creative Commons Attribution (CC BY-NC) license (https://creativecommons.org/licenses/by-nc/4.0/). See http://ivyspring.com/terms for full terms and conditions.

Received: 2018.03.14; Accepted: 2018.07.03; Published: 2018.07.27

\begin{abstract}
Background \& aims: VEGFR-3 has been shown of great significance in lymph node metastasis and some malignancies, however, its expression in tumors and impact on outcome of intrahepatic cholangiocarcinoma (iCCA) remains unknown. The aim of this study was to assess the role of VEGFR-3 positive tumors for prognosis of iCCA and tumor-associated lymphangiogenesis.

Methods: Clinicopathological features, prognostic factors and survival rate were analyzed to evaluate the influence of VEGFR-3 positive expression on prognosis of iCCA. In addition, tumor-associated lymphangiogenesis quantified as micro-lymphatic vessel density (MLVD) was assessed to explore the correlation between VEGFR-3 expression and lymph node metastasis for iCCA.

Results: Patients with VEGFR-3 positive tumors had increased lymph node metastasis $(p=0.025)$ and were more likely to suffer from tumor recurrence compared with VEGFR-3 negative tumors $(p<0.001)$. VEGFR-3 expression in tumors was identified as an independent prognostic factor for both overall and recurrence-free survival in surgical resected patients with iCCA. In addition, higher MLVD was significantly associated with VEGFR-3 positive expression in tumors $(p<0.001)$, which facilitate lymph node metastasis and significantly worse survival rates.

Conclusions: Our study reveals that VEGFR-3 positive expression in tumors represents an independent prognostic factor for both overall and recurrence-free survival in hepatic resected patients with iCCA. VEGFR-3 positive tumors favor lymph node metastasis, tumor recurrence and worse outcomes through tumor-associated lymphangiogenesis.
\end{abstract}

Key words: VEGFR-3 expression, intrahepatic cholangiocarcinoma, lymph node metastasis, tumor-associated lymphangiogenesis, prognosis

\section{Introduction}

Intrahepatic cholangiocarcinoma (iCCA), the second most common primary liver cancer after hepatocellular carcinoma, has been shown a drastic increase in the incidence and mortality [1, 2]. It is a highly malignant tumor with poor prognosis due to rapid progression, lymph node metastasis and frequent recurrence of the tumor. Surgical resection of iCCA provides the potential curative treatment that prolongs survival outcomes; however, most patients present with unresectable iCCA at the time of diagnosis due to lack of early clinical manifestation and advanced cancer cells or metastasis [3, 4]. Moreover, the efficacy of chemotherapy or radiotherapy to provide better survival outcomes is not confirmed $[5,6]$. Therefore, in consideration of increasing number of patients and limited therapeutic strategies, novel molecular markers of iCCA are required for prognosis prediction and therapeutic target. 
The tyrosine kinase receptor, vascular endothelial growth factor receptor-3 (VEGFR-3), binds different ligands, including VEGFC and VEGFD, which initiate intracellular signaling cascades leading to lymphatic vascular sprouting and development [7]. The VEGFC/VEGFD-VEGFR-3 axis has also been discovered to promote tumor-associated lymphangiogenesis and metastasis in experimental tumor models [8, 9]. Using neutralizing VEGFR-3-specific monoclonal antibodies result in a restriction of tumor lymphangiogenesis and lymph node metastasis [10, 11]. Recently, VEGFR-3 is also found to be expressed in a wide variety of malignancies and correlated with worse survival, including breast cancer [12], non-small-cell lung cancer [13], endometrial carcinoma [14], etc.

Although VEGFR-3 is expressed in several types of tumors and contributes to lymphangiogenesis in animal models, the role of VEGFR-3 expression in human iCCA and the correlation between VEGFR-3 expression and tumor-associated lymphangiogenesis has not been studied. In this study, we investigated the expression of VEGFR-3 in tumors and its correlation with clinicopathological feature of iCCA. The association between VEGFR-3 expression and tumor-associated lymphangiogenesis and survival analysis of postoperative patients were also evaluated to investigate the prognostic significance of VEGFR-3 expression in tumors.

\section{Patients and Methods}

\section{Study patients}

From Jan 2007 to Jul 2015 in our institution, a total of 106 consecutive patients who underwent hepatic resection with curative intent for iCCA were enrolled in the study. The included patients met the criteria as follows, (1) patients with pathological confirmation of iCCA from liver specimens, (2) patients who received no preoperative adjuvant chemotherapy or radiation therapy, (3) patients without distant metastasis. The present study was approved by the local ethics committee of Renji Hospital, School of Medicine, Shanghai Jiao Tong University.

\section{Data collection}

Preoperative evaluation of the patients, including demographic data, serological examinations, and imaging studies was performed routinely. Regional lymph node dissection was performed in all patients. Lymph node metastasis and vascular involvement were evaluated based on preoperative imaging studies, intraoperative exploration, and postoperative pathology.
All patients were followed up in the outpatient at regular intervals, and those who were with unavailable data were visited by telephone inquiries or dropping-in follow-up. The primary endpoint of this study was death of patients, and the second endpoint was recurrence of the tumor. Data of last follow-up investigation and recurrence of the tumor were collected for all patients.

\section{Immunohistochemistry}

4-micrometer thick formalin-fixed, paraffinembedded sections were cut and deparaffinized through xylene and ethanol. Methanol containing $0.3 \%$ hydrogen peroxidase was used to block the endogenous peroxidase activity. After antigen retrieval and washing in phosphate-buffered saline, tissue sections were incubated overnight at $4^{\circ} \mathrm{C}$ with the monoclonal antibodies against VEGFR-3 (rabbit monoclonal, 1:200; Abcam) and podoplanin (rabbit monoclonal, 1:200; Abcam). On the second day, the sections were then incubated with the appropriate biotinylated secondary antibodies and the immune complex was then visualized using 3,3'-diaminobenzidine.

\section{Assessment of VEGFR-3 expression in cancer cells}

To analyze VEGFR-3 expression in cancer cells, percentage of immunostained cancer cells and staining intensity were assessed semi-quantitatively. For each section, three fields were randomly selected and staining in the cytoplasm was considered positively immunostained. The tissue sections were scored based on the percentage of immunostained cancer cells as: $<10 \%=0,10-50 \%=1,>50 \%=2$. Sections were also scored on basis of staining intensity as negative $=0$, moderate $=1$, or strong $=2$. A final score was obtained by multiplying the intensity and percentage scores. Expression of VEGFR-3 in cancer cells was categorized as either positive $(\geq 2)$ or negative $(<2)$ using a cut-off score of 2 . All sections were evaluated independently by two investigators, who were blinded for the histopathological characteristics of the tumors and the patients.

\section{Assessment of micro-lymphatic vessel density (MLVD)}

To analyze tumor-associated lymphangiogenesis, micro-lymphatic vessel density (MLVD) was determined in the tumor sections by quantitative analysis of podoplanin-positive vessels. The most vascularized areas within tumor (hot spot) were identified at low magnification $(\times 40)$ and were manually counted at a representative high magnification $(\times 400)$. The lymphatic vessels were 
counted in three fields and were recorded as total number per unit area. Endothelial staining in large vessels and non-endothelial structures were disregarded in lymphatic vessel counts.

\section{Statistical analysis}

The correlation between VEGFR-3 category and the clinicopathologic factors were evaluated using the chi-square test. Survival curves were estimated using the Kaplan-Meier method, and difference was analyzed using the log-rank test. KolmogorovSmirnov- and Shapiro-Wilk-test were used to test MLVD values for normal distribution. Non-parametric data were compared using the Mann-Whitney-U-test. MLVD was analyzed by median cut-off and box-scatter plot graphics display a statistical summary of the median, quartiles and ranges. To evaluate independent prognostic factors associated with survival, the Cox regression hazard model was used to perform univariate and multivariate analysis. All statistical analysis was performed using software (SPSS, version 19.0). Statistical significance was defined as $p<0.05\left({ }^{*} p<\right.$ $0.05 ;{ }^{* *} p<0.01 ;{ }^{* * *} p<0.001$ ).

\section{Results}

\section{Clinicopathological characteristics}

The clinicopathological characteristics of the 106 patients were described in Supp. Table 1. All patients had undergone hepatic resection and none received preoperative adjuvant chemotherapy or radiation therapy. Carbohydrate antigen 19-9 (CA19-9), one of the tumor markers for iCCA, was above the normal level in majority of patients $(n=73,68.9 \%)$. During the operation, presence of vascular invasion, lymph node metastasis and perineural sheath infiltration, which represented tumor progression, were found in 28,48 , 14 patients, respectively. In addition, 61 patients $(57.5 \%)$ presented with largest tumor diameter over than $50 \mathrm{~mm}$ and 17 patients $(16 \%)$ were with multiple tumor nodes. The median follow-up of total patients was 22.1 months (range, 1-118 months). Tumor recurrence occurred in 72 patients by the end of follow-up.

\section{Expression of VEGFR-3 in ICCA}

VEGFR-3 expression was detected within the tumors exhibiting in the cytoplasm of cancer cells in 106 iCCA samples. The staining intensity of VEGFR-3 varied between individuals with iCCA (Fig. 1A, B, C, $\mathrm{D}, \mathrm{E}, \mathrm{F})$, thus, in order to analyze the impact of VEGFR-3 expression on outcomes and prognosis, patients were divided into two groups according to quantitative analysis by multiplying the intensity and percentage scores. Of 106 patients enrolled in the study, 77 displayed VEGFR-3 expression score $\geq 2$ and were assigned to the "positive" group, while the other 29 patients were allocated to the "negative" group (Fig. 1G).

\section{Correlation between VEGFR-3 expression and clinicopathogical features in iCCA}

The correlation between the VEGFR-3 expression and clinicopathological characteristics was statistically analyzed in Table 1 . Patients with VEGFR-3-positive tumors showed more tumor numbers (16 out of 77 vs. 1 out of 29, $p=0.030$ ) and more frequently lymph node metastasis (40 out of 77 vs. 8 out of $29, p=0.025)$ compared with VEGFR-3-negative tumors. Moreover, patients with VEGFR-3-positive tumors developed tumor recurrence more frequently than patients with VEGFR-3 negative tumors (60 out of 77 vs. 12 out of 29, $p<0.001$ ) (Fig. 2A). Other clinicopathological parameters, including gender, HBV infection, preoperative AFP and CA19-9 level, tumor size, distribution, vascular invasion, histological differentiation and perineural sheath infiltration were not significantly different between both groups (Supp. Fig. 1).

Table 1. Correlation between VEGFR-3 expression by cancer cells and clinicopathological characteristics in patients with iCCA

\begin{tabular}{|c|c|c|c|}
\hline \multirow{2}{*}{$\begin{array}{l}\text { Clinicopathological } \\
\text { Characteristics }\end{array}$} & \multicolumn{3}{|c|}{ VEGFR-3 expression by cancer cells } \\
\hline & Negative & Positive & $p$ value \\
\hline No. of patients & 29 & 77 & \\
\hline \multicolumn{4}{|l|}{ Age (years) } \\
\hline$<60$ & $20(62.5 \%)$ & $36(42.0 \%)$ & 0.041 \\
\hline$\geq 60$ & $9(37.5 \%)$ & $41(58.0 \%)$ & \\
\hline \multicolumn{4}{|l|}{ Gender } \\
\hline Male & $13(53.6 \%)$ & $31(64.0 \%)$ & 0.670 \\
\hline Female & $16(46.4 \%)$ & $46(36.0 \%)$ & \\
\hline \multicolumn{4}{|l|}{ HBV infection } \\
\hline Absent & $17(48.2 \%)$ & $47(74.0 \%)$ & 0.820 \\
\hline Present & $12(51.8 \%)$ & $30(26.0 \%)$ & \\
\hline \multicolumn{4}{|c|}{ Preoperative AFP $(\mu \mathrm{g} / \mathrm{l})$} \\
\hline$<9$ & $18(71.4 \%)$ & $60(76.0 \%)$ & 0.099 \\
\hline$\geq 9$ & $11(28.6 \%)$ & $17(24.0 \%)$ & \\
\hline \multicolumn{4}{|l|}{$\begin{array}{l}\text { Preoperative CA19-9 } \\
(\mathrm{U} / \mathrm{ml})\end{array}$} \\
\hline$<35$ & $11(33.9 \%)$ & $22(28.0 \%)$ & 0.354 \\
\hline$\geq 35$ & $18(66.1 \%)$ & $55(72.0 \%)$ & \\
\hline \multicolumn{4}{|l|}{ Tumor size (mm) } \\
\hline$<50$ & $13(48.2 \%)$ & $32(36.0 \%)$ & 0.761 \\
\hline$\geq 50$ & $16(51.8 \%)$ & $45(64.0 \%)$ & \\
\hline \multicolumn{4}{|l|}{ Tumor number } \\
\hline Single & $28(89.3 \%)$ & $61(78.0 \%)$ & 0.030 \\
\hline Multiple & $1(10.7 \%)$ & $16(22.0 \%)$ & \\
\hline \multicolumn{4}{|l|}{ Vascular invasion } \\
\hline Absent & $24(73.2 \%)$ & $54(74.0 \%)$ & 0.189 \\
\hline Present & $5(26.8 \%)$ & $23(26.0 \%)$ & \\
\hline \multicolumn{4}{|l|}{ Lymph node metastasis } \\
\hline Absent & $21(71.4 \%)$ & $37(36.0 \%)$ & 0.025 \\
\hline Present & $8(28.6 \%)$ & $40(64.0 \%)$ & \\
\hline \multicolumn{4}{|c|}{ Histologic differentiation } \\
\hline Well or moderate & $12(51.8 \%)$ & $40(46.0 \%)$ & 0.332 \\
\hline Poor & $17(48.2 \%)$ & $37(54.0 \%)$ & \\
\hline \multicolumn{4}{|l|}{ Distribution } \\
\hline Unilobar & $11(42.9 \%)$ & $30(34.0 \%)$ & 0.923 \\
\hline
\end{tabular}




\begin{tabular}{|c|c|c|c|}
\hline \multirow{2}{*}{$\begin{array}{l}\text { Clinicopathological } \\
\text { Characteristics }\end{array}$} & \multicolumn{3}{|c|}{ VEGFR-3 expression by cancer cells } \\
\hline & Negative & Positive & $p$ value \\
\hline Bilobar & $18(57.1 \%)$ & $47(66.0 \%)$ & \\
\hline \multicolumn{4}{|l|}{$\begin{array}{l}\text { Perineural sheath } \\
\text { infiltration }\end{array}$} \\
\hline Absent & $27(87.5 \%)$ & $65(86.0 \%)$ & 0.239 \\
\hline Present & $2(12.5 \%)$ & $12(14.0 \%)$ & \\
\hline \multicolumn{4}{|l|}{ Tumor recurrence } \\
\hline Absent & $17(58.9 \%)$ & $17(2.0 \%)$ & $<0.001$ \\
\hline Present & $12(41.1 \%)$ & $60(98.0 \%)$ & \\
\hline
\end{tabular}

\section{Analysis of prognostic factors in patients with iCCA}

Univariate and multivariate analyses were performed to assess the independent prognostic factors for overall and recurrence-free survival (Table $2,3)$. For overall survival, univariate analysis showed that the significant prognostic factors included tumor size $(p=0.003)$, lymph node metastasis $(p<0.001)$ and VEGFR-3 expression in tumors $(p<0.001)$. In the multivariate analysis, tumor size $(p=0.004)$, lymph node metastasis $(p<0.001)$ and VEGFR-3 expression $(p=0.002)$ were all identified as independent prognostic factors for overall survival (Fig. 2B). For recurrence-free survival, the above 3 factors included tumor size $(p=0.001)$, lymph node metastasis $(p=0.010)$ and VEGFR-3 expression $(p=0.006)$ were also determined as independent risk factors through univariate and multivariate analyses. In addition, preoperative HBV infection $(p=0.011)$ also serve as one of the independent prognostic factors for recurrence-free survival (Fig. 2C). It is noted that neither intensity nor percentage of VEGFR-3 staining was prognostic factors for survivals.

\section{Impact of VEGFR-3 expression on survival in iCCA}

Since VEGFR-3 expression in tumors revealed independent prognostic significance for both overall and recurrence-free survival, the influence of VEGFR-3 expression on survival in iCCA was analyzed (Fig. 2D \& 2E). The 1-, 3-, and 5-year survival of patients with VEGFR-3 positive tumors $(\mathrm{n}=77)$ was $40.3 \%, 28.3 \%$ and $14.6 \%$, respectively, which was much worse than patients with VEGFR-negative tumors displaying 1-, 3-, and 5-year survival of $75.9 \%, 65.3 \%$ and $53.2 \%$, respectively $(p<0.001)$. Similarly, patients with VEGFR-3 positive
A

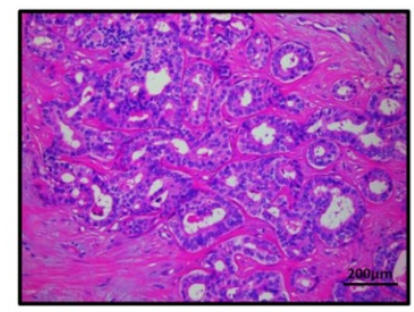

D

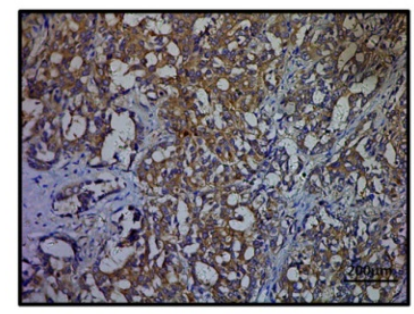

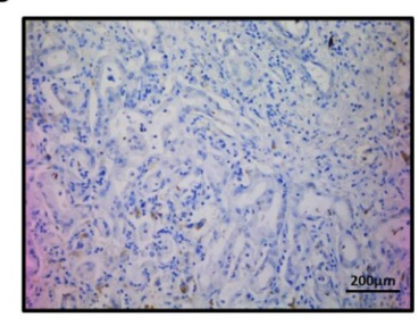

E

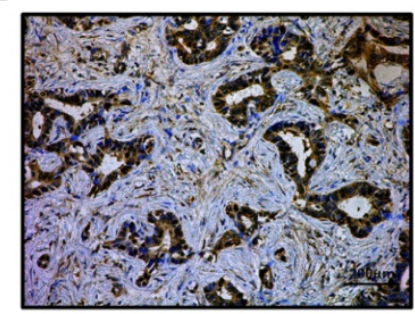

C

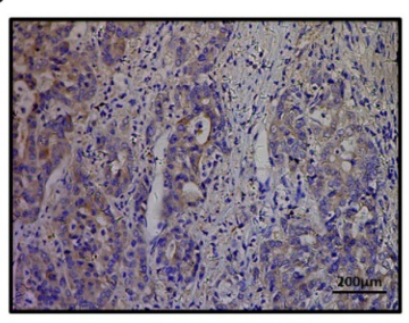

$\mathbf{F}$

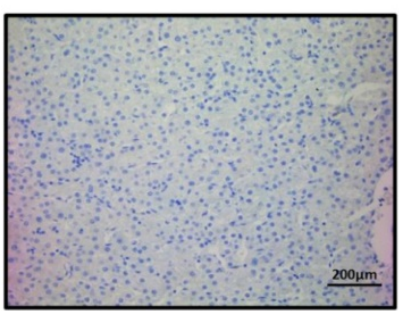

G
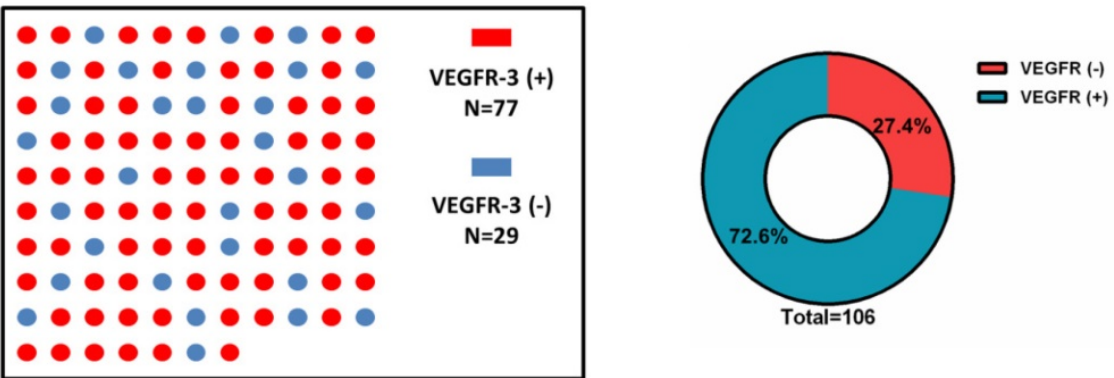

Figure 1. Different expression of VEGFR-3 in intrahepatic cholangiocarcinoma (iCCA). (A) Representative iCCA tissue characterized by a glandular architectural pattern (hematein-eosin-saffron staining). (B) Negative, (C) Weak, (D) Moderate and (E) Strong immunostaining intensity for VEGFR-3 in cancer cells of iCCA tissue. Strong staining intensity for VEGFR-3 in the cytoplasm of cancer cells was regarded as positive controls. (F) Staining for VEGFR-3 was negative in all para-carcinoma tissues and was regarded as negative controls. (G) Of total 106 patients enrolled in the study, 77 patients $(72.6 \%)$ with iCCA showed positive expression of VEGFR-3 in tumors, while 29 patients (27.4\%) presented with negative expression. (scale bar: $200 \mu \mathrm{m}$ ) 
A

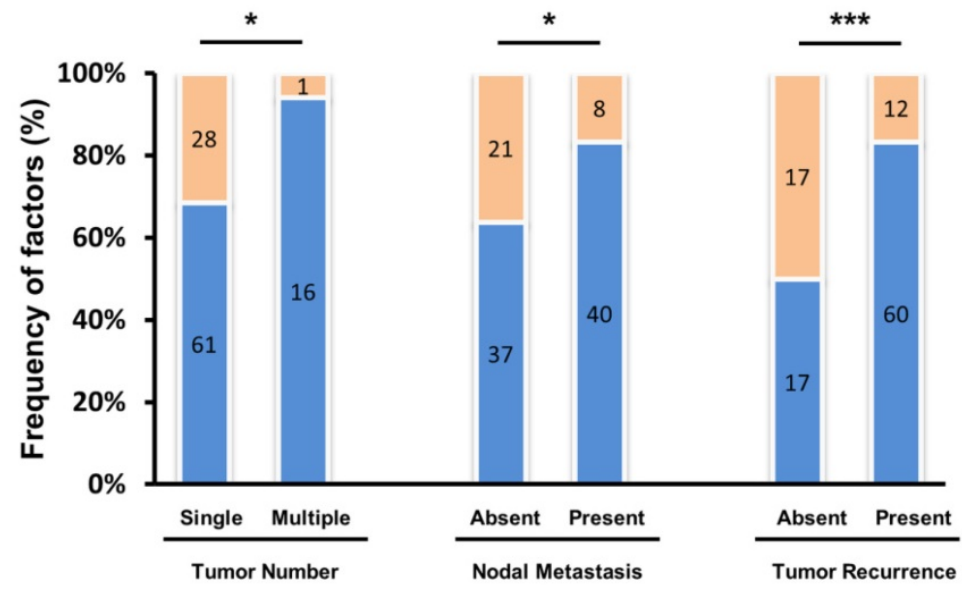

VEGFR-3 (-) tumors

VEGFR-3 (+) tumors

B

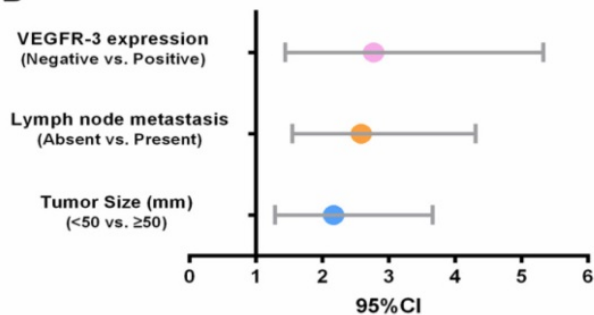

D

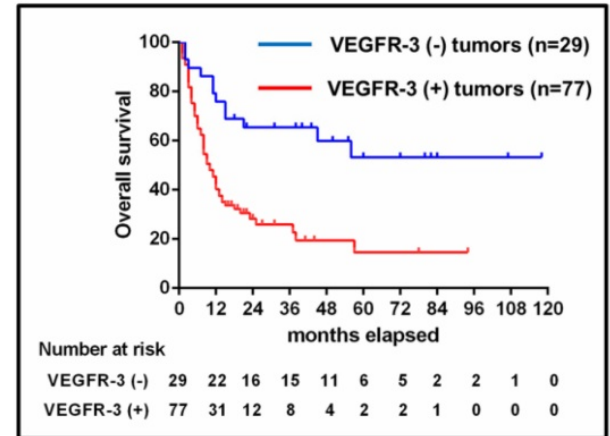

C

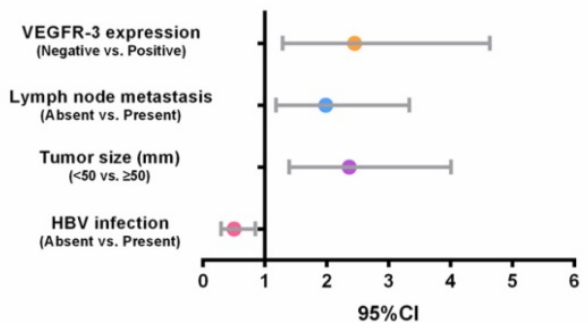

E

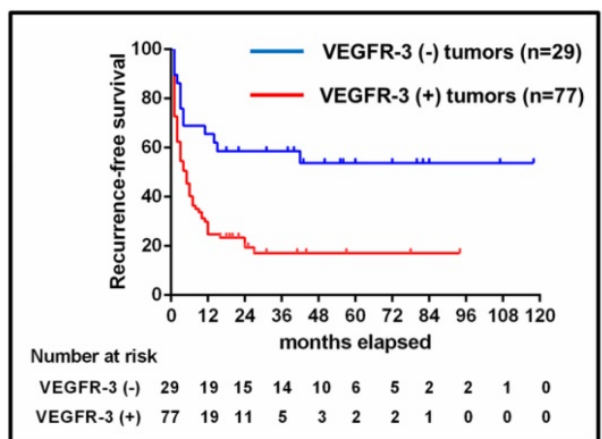

Figure 2. Impact of VEGFR-3 expression on survivals and clinicopathological characteristics and its clinical significance in patients with iCCA. (A) Patients with VEGFR-3-positive tumors showed more frequently multiple tumor numbers (chi-square-test, $p=0.030$ ), lymph node metastasis (chi-square-test, $p=0.025)$ and tumor recurrence (chi-square-test, $p<0.001)$ compared with VEGFR-3-negative tumors. (B) VEGFR-3 expression $(95 \% \mathrm{Cl}$ : 1.445-5.330, $p=0.002)$, tumor size $(95 \% \mathrm{Cl}$ : $1.287-3.661$, $p=0.004)$ and lymph node metastasis $(95 \% \mathrm{Cl}: 1.552-4.311, p<0.001)$ were identified as independent prognostic factors for overall survival in patients with iCCA. $(C)$ For recurrence survival, VEGFR-3 expression ( $95 \% \mathrm{Cl}$ : $1.293-4.640, p=0.006)$, tumor size $(95 \% \mathrm{Cl}: 1.396-4.009, p=0.001)$ and lymph node metastasis $(95 \% \mathrm{Cl}$ : $1.181-3.336, p=0.010)$ also revealed independent prognostic significance. In addition, $\mathrm{HBV}$ infection $(95 \% \mathrm{Cl}$ : $0.299-0.852, p=0.011)$ served as the independent positive factor for recurrence-free survival. (D) The overall survival rates of hepatic resected patients of iCCA with VEGFR-3 positive tumors ( $n=77$ ) were much worse than those of patients with VEGFR-3 negative tumors $(n=29)(p<0.001)$. (E) Patients with VEGFR-3 positive tumors $(n=77)$ also had much worse recurrence-free survival compared with those of patients with VEGFR-3 negative tumors $(n=29)(p<0.001)$.

tumors were more likely to suffer from tumor recurrence, with 1-, 3-, and 5-year recurrence-free survival of $24.7 \%, 19.5 \%$ and $17 \%$, respectively, as compared to $65.5 \%, 58.6 \%$ and $53.7 \%$, respectively, in patients with VEGFR-3 negative tumors.

\section{Correlation between micro-lymphatic vessel density (MLVD) and clinicopathological parameters}

To determine whether lymphangiogenesis translates characteristics, we performed a quantitative analysis as shown in Table 4. The proportion of micro-lymphatic vessel density (MLVD) was higher in the cases with absence of HBV infection, multiple tumor nodes, presence of lymph node metastasis and tumor recurrence $(p=0.002,0.009,<0.001$ and $<0.001$, respectively) (Fig. 3E). Moreover, the higher MLVD was significantly associated with VEGFR-3 positive expression in tumors $(p<0.001)$ (Fig. 3A, B, C, D); meanwhile, other clinicopathological parameters showed no significant correlation with 
tumor-associated lymphangiogenesis (Supp. Fig. 2). Further, to avoid bias of patient selection, the correlation between MLVD and clinicopathological features was analyzed in 77 patients with VEGFR-3 positive tumors (Supp. Table 2). It was shown that in patients with VEGFR-3 positive tumors, the level of MLVD was still significantly higher in individuals with absence of HBV infection, lymph node metastasis and tumor recurrence $(p=0.002,0.003$ and $<0.001$, respectively) (Fig. 4C), while other clinicopathological parameters revealed no significant correlation with the proliferative rate of tumor-associated lymphangiogenesis (Supp. Fig. 3).

Table 2. Univariate and multivariate analysis of prognosis factors for overall survival of hepatic resected patients with iCCA

\begin{tabular}{|c|c|c|c|c|c|c|}
\hline \multirow[t]{2}{*}{ Variable } & \multicolumn{3}{|c|}{ Univariate analysis } & \multicolumn{3}{|c|}{ Multivariate analysis } \\
\hline & HR & $95 \% \mathrm{CI}$ & $\begin{array}{l}p \\
\text { value }\end{array}$ & HR & $95 \% \mathrm{CI}$ & $\begin{array}{l}p \\
\text { value }\end{array}$ \\
\hline Age (<60 vs. $\geq 60)$ & 1.226 & $0.767-1.961$ & 0.394 & - & - & - \\
\hline Gender (Male vs. Female) & 1.564 & $0.957-2.554$ & 0.074 & - & - & - \\
\hline $\begin{array}{l}\text { HBV infection (Absent vs. } \\
\text { Present) }\end{array}$ & 0.674 & $0.411-1.106$ & 0.118 & - & - & - \\
\hline $\begin{array}{l}\text { Preoperative AFP }(\mu \mathrm{g} / \mathrm{l})(<9 \\
\text { vs. } \geq 9)\end{array}$ & 0.814 & $0.469-1.414$ & 0.466 & - & - & - \\
\hline $\begin{array}{l}\text { Preoperative CA19-9 (U/ml) } \\
(<35 \text { vs. } \geq 35)\end{array}$ & 1.647 & $0.952-2.849$ & 0.075 & - & - & - \\
\hline Tumor size $(\mathrm{mm})(<50$ vs. $\geq 50)$ & 2.183 & $1.312-3.631$ & 0.003 & 2.170 & $1.287-3.661$ & 0.004 \\
\hline $\begin{array}{l}\text { Vascular invasion (Absent vs. } \\
\text { Present) }\end{array}$ & 1.622 & $0.976-2.695$ & 0.062 & - & - & - \\
\hline $\begin{array}{l}\text { Lymph node metastasis } \\
\text { (Absent vs. Present) }\end{array}$ & 3.161 & $1.916-5.215$ & $<0.001$ & 2.587 & $1.552-4.311$ & $<0.001$ \\
\hline $\begin{array}{l}\text { Tumor number (Single vs. } \\
\text { Multiple) }\end{array}$ & 1.468 & $0.815-2.646$ & 0.201 & - & - & - \\
\hline $\begin{array}{l}\text { Histologic differentiation } \\
\text { (Well/Moderate vs. Poor) }\end{array}$ & 1.149 & $0.719-1.838$ & 0.561 & - & - & - \\
\hline $\begin{array}{l}\text { Perineural sheath infiltration } \\
\text { (Absent vs. Present) }\end{array}$ & 1.188 & $0.608-2.321$ & 0.615 & - & - & - \\
\hline $\begin{array}{l}\text { Distribution (Unilobar vs. } \\
\text { Bilobar) }\end{array}$ & 1.482 & $0.894-2.457$ & 0.127 & - & - & - \\
\hline $\begin{array}{l}\text { Percentage of VEGFR-3 } \\
\text { staining } \\
(<10 \% \text { vs. } 10-50 \% \text { vs. }>50 \%)\end{array}$ & 1.322 & $0.938-1.864$ & 0.111 & & & \\
\hline $\begin{array}{l}\text { Intensity of VEGFR-3 staining } \\
\text { (negative vs. moderate vs. } \\
\text { strong) }\end{array}$ & 1.364 & $0.949-1.961$ & 0.094 & & & \\
\hline $\begin{array}{l}\text { VEGFR-3 expression (Negative } \\
\text { vs. Positive) }\end{array}$ & 3.099 & $1.640-5.854$ & $<0.001$ & 2.775 & $1.445-5.330$ & 0.002 \\
\hline
\end{tabular}

Table 3. Univariate and multivariate analysis of prognosis factors for recurrence-free survival of hepatic resected patients with iCCA

\begin{tabular}{|c|c|c|c|c|c|c|}
\hline \multirow[t]{2}{*}{ Variable } & \multicolumn{3}{|c|}{ Univariate analysis } & \multicolumn{3}{|c|}{ Multivariate analysis } \\
\hline & HR & 95\%CI & $\begin{array}{l}p \\
\text { value }\end{array}$ & HR & $95 \% \mathrm{CI}$ & $\begin{array}{l}p \\
\text { value }\end{array}$ \\
\hline Age (<60 vs. $\geq 60)$ & 1.302 & $0.827-2.049$ & 0.254 & - & - & - \\
\hline Gender (Male vs. Female) & 1.129 & $0.711-1.793$ & 0.608 & - & - & - \\
\hline $\begin{array}{l}\text { HBV infection (Absent vs. } \\
\text { Present) }\end{array}$ & 0.520 & $0.319-0.847$ & 0.009 & 0.505 & $0.299-0.852$ & 0.011 \\
\hline $\begin{array}{l}\text { Preoperative AFP }(\mu \mathrm{g} / \mathrm{l})(<9 \text { vs. } \\
\geq 9)\end{array}$ & 0.889 & $0.527-1.499$ & 0.658 & - & - & - \\
\hline $\begin{array}{l}\text { Preoperative CA19-9 }(\mathrm{U} / \mathrm{ml}) \\
(<35 \text { vs. } \geq 35)\end{array}$ & 1.499 & $0.897-2.504$ & 0.122 & - & - & - \\
\hline Tumor size $(\mathrm{mm})(<50 \mathrm{vs} . \geq 50)$ & 2.044 & $1.258-3.321$ & 0.004 & 2.366 & $1.396-4.009$ & 0.001 \\
\hline $\begin{array}{l}\text { Vascular invasion (Absent vs. } \\
\text { Present) }\end{array}$ & 1.286 & $0.782-2.116$ & 0.322 & - & - & - \\
\hline $\begin{array}{l}\text { Lymph node metastasis } \\
\text { (Absent vs. Present) }\end{array}$ & 2.816 & $1.747-4.538$ & $<0.001$ & 1.985 & $1.181-3.336$ & 0.010 \\
\hline
\end{tabular}

\begin{tabular}{|c|c|c|c|c|c|c|}
\hline \multirow[t]{2}{*}{ Variable } & \multicolumn{3}{|c|}{ Univariate analysis } & \multicolumn{3}{|c|}{ Multivariate analysis } \\
\hline & HR & $95 \% \mathrm{CI}$ & $\begin{array}{l}p \\
\text { value }\end{array}$ & HR & $95 \% \mathrm{CI}$ & $\begin{array}{l}p \\
\text { value }\end{array}$ \\
\hline $\begin{array}{l}\text { Tumor number (Single vs. } \\
\text { Multiple) }\end{array}$ & 1.752 & $1.002-3.064$ & 0.049 & 0.871 & $0.477-1.593$ & 0.655 \\
\hline $\begin{array}{l}\text { Histologic differentiation } \\
\text { (Well/Moderate vs. Poor) }\end{array}$ & 1.084 & $0.689-1.706$ & 0.727 & - & - & - \\
\hline $\begin{array}{l}\text { Perineural sheath infiltration } \\
\text { (Absent vs. Present) }\end{array}$ & 1.017 & $0.522-1.980$ & 0.960 & - & - & - \\
\hline $\begin{array}{l}\text { Distribution (Unilobar vs. } \\
\text { Bilobar) }\end{array}$ & 1.402 & $0.867-2.269$ & 0.169 & - & - & - \\
\hline $\begin{array}{l}\text { Percentage of VEGFR-3 } \\
\text { staining } \\
(<10 \% \text { vs. } 10-50 \% \text { vs. }>50 \%)\end{array}$ & 1.348 & $0.959-1.894$ & 0.086 & & & \\
\hline $\begin{array}{l}\text { Intensity of VEGFR-3 staining } \\
\text { (negative vs. moderate vs. } \\
\text { strong) }\end{array}$ & 1.259 & $0.887-1.787$ & 0.198 & & & \\
\hline $\begin{array}{l}\text { VEGFR-3 expression (Negative } \\
\text { vs. Positive) }\end{array}$ & 2.717 & $1.480-4.990$ & 0.001 & 2.450 & $1.293-4.640$ & 0.006 \\
\hline
\end{tabular}

Table 4. Correlation of micro-lymphatic vessel density (MLVD) with clinicopathological characteristics in 106 patients with iCCA

\begin{tabular}{|c|c|c|c|}
\hline Variable & Cases & MLVD (median (IQR)) & $p$ value \\
\hline \multicolumn{4}{|l|}{ Age } \\
\hline$<60$ & 56 & $9.5(7-16)$ & 0.060 \\
\hline$\geq 60$ & 50 & $13.5(7.75-20)$ & \\
\hline \multicolumn{4}{|l|}{ Gender } \\
\hline Male & 44 & $11(6.25-18)$ & 0.525 \\
\hline Female & 62 & $13(7-18)$ & \\
\hline \multicolumn{4}{|l|}{ HBV infection } \\
\hline Absent & 64 & $14(8.25-19.75)$ & 0.002 \\
\hline Present & 42 & $8.5(6-15.25)$ & \\
\hline \multicolumn{4}{|c|}{ Preoperative AFP $(\mu \mathrm{g} / \mathrm{l})$} \\
\hline$<9$ & 78 & $12(7-18)$ & 0.417 \\
\hline$\geq 9$ & 28 & $9.5(7-18.25)$ & \\
\hline \multicolumn{4}{|c|}{ Preoperative CA19-9 (U/ml) } \\
\hline$<35$ & 33 & $12(6.5-17)$ & 0.523 \\
\hline$\geq 35$ & 73 & $12(7-18)$ & \\
\hline \multicolumn{4}{|l|}{ Tumor size (mm) } \\
\hline$<50$ & 45 & $9(6-16)$ & 0.053 \\
\hline$\geq 50$ & 61 & $13(8-18.5)$ & \\
\hline \multicolumn{4}{|l|}{ Tumor number } \\
\hline Single & 89 & $11(6.5-17.5)$ & 0.009 \\
\hline Multiple & 17 & $17(10.5-21)$ & \\
\hline \multicolumn{4}{|l|}{ Vascular invasion } \\
\hline Absent & 78 & $12(7-18)$ & 0.605 \\
\hline Present & 28 & $11.5(8-18.75)$ & \\
\hline \multicolumn{4}{|c|}{ Lymph node metastasis } \\
\hline Absent & 58 & $8(6-15.25)$ & $<0.001$ \\
\hline Present & 48 & $15(10.25-20)$ & \\
\hline \multicolumn{4}{|c|}{ Histologic differentiation } \\
\hline Well or moderate & 52 & $10(6-19)$ & 0.653 \\
\hline Poor & 54 & $12.5(7.75-17.25)$ & \\
\hline \multicolumn{4}{|l|}{ Distribution } \\
\hline Unilobar & 41 & $10(6-19)$ & 0.163 \\
\hline Bilobar & 65 & $12.5(7.75-17.25)$ & \\
\hline \multicolumn{4}{|c|}{ Perineural sheath infiltration } \\
\hline Absent & 92 & $12(7-18)$ & 0.562 \\
\hline Present & 14 & $13(8-19)$ & \\
\hline \multicolumn{4}{|l|}{ Tumor recurrence } \\
\hline Absent & 34 & $6(5-7.25)$ & $<0.001$ \\
\hline Present & 72 & $16(11.25-20)$ & \\
\hline \multicolumn{4}{|c|}{ VEGFR-3 expression } \\
\hline Negative & 29 & $6(5-8)$ & $<0.001$ \\
\hline Positive & 77 & $15(9-19.5)$ & \\
\hline
\end{tabular}

\section{Analysis of prognostic factors in iCCA patients with VEGFR-3 positive tumors}

As shown in Supp. Table 3 and 4, further assessment of independent risk factors for survival in 77 patients with VEGFR-3 positive tumors was 
performed. For overall survival, both tumor size $(p=0.045)$ and MLVD analyzed by interquartile range $(p<0.001)$ were identified as independent prognostic factors (Fig. 4A). MLVD analyzed by interquartile range $(p<0.001)$ also served prognostic significance for recurrence-free survival in patients with VEGFR-3 positive tumors (Fig. 4B), while, other clinicopathological parameters revealed no independent prognostic significance for survival.
A

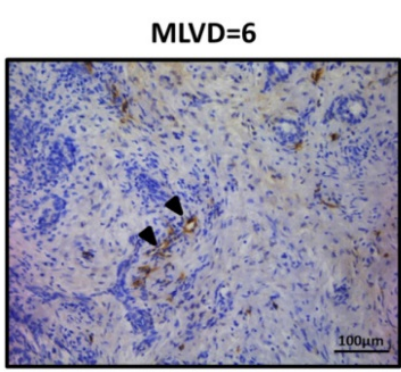

D

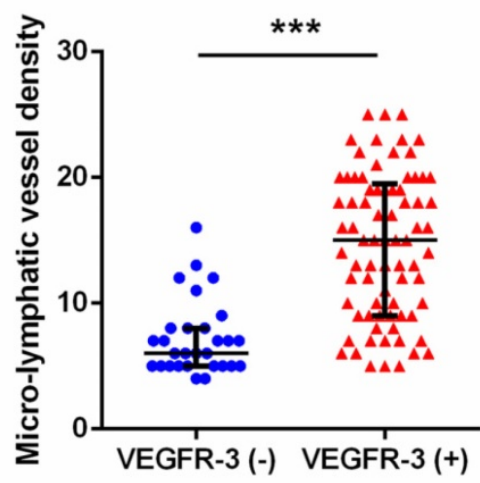

B

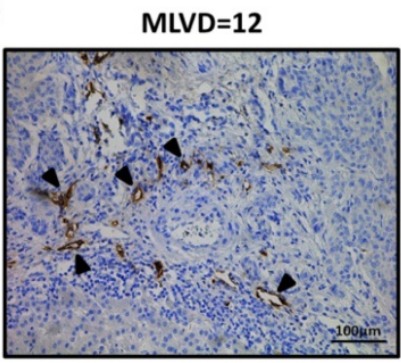

C

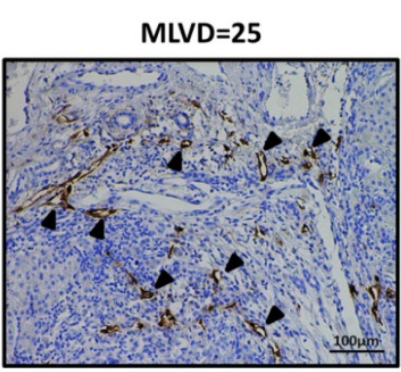

E

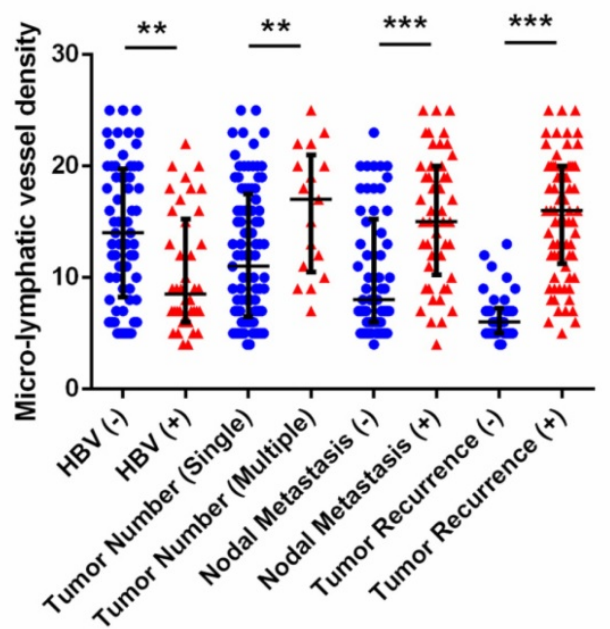

Figure 3. Positive expression of VEGFR-3 correlates with tumor-associated lymphangiogenesis in iCCA. Representative tissue sections of iCCA showed low $(M L V D=6)(A)$, median $(M L V D=12)(B)$ and high $(M L V D=25)(C)$ level of tumor-associated lymphangiogenesis assessed by micro-lymphatic vessel density (MLVD). (arrows, micro-lymphatic vessels; scale bar $=100 \mu \mathrm{m}$ ). (D) In all 106 hepatic resected patients with iCCA, patients with VEGFR-3 positive tumors had significantly higher proportion of MLVD (U-test, $p<0.001)$. (E) Higher level of MLVD was also closely correlated with negative HBV infection, multiple tumor numbers, lymph node metastasis and tumor recurrence (U-test, $p=0.002,0.009,<0.001$ and $<0.001$, respectively). Box-scatter plot graphics display a statistical summary of the median, quartiles and ranges of MLVD.

A

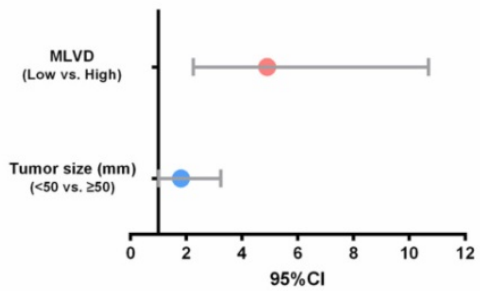

B

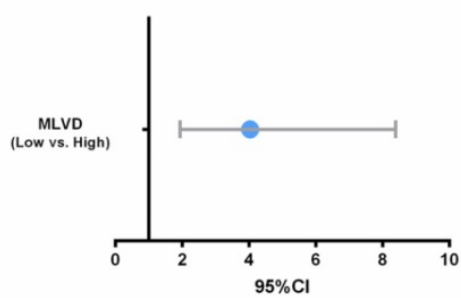

C

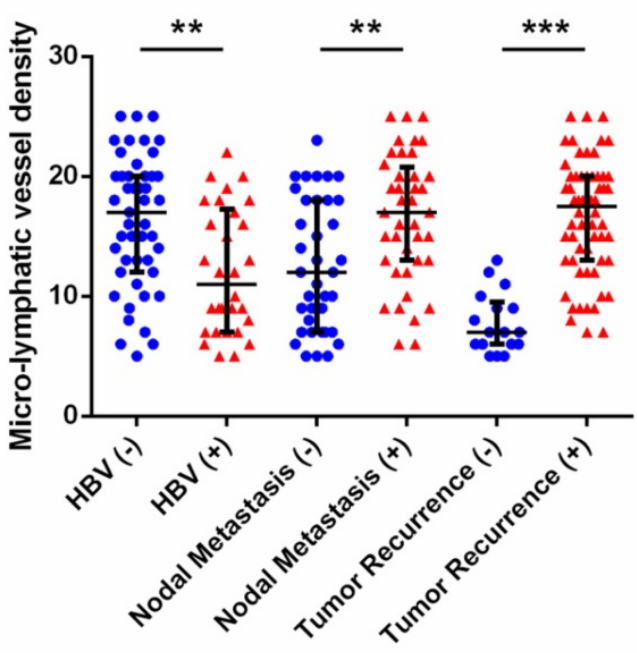

Figure 4. Prognostic factors and MLVD in 77 patients with VEGFR-3 positive tumors. (A) For overall survival, tumor size $(95 \% \mathrm{Cl}$ : $1.013-3.245$, $p=0.045)$ and $M L V D$ analyzed by interquartile range $(95 \% \mathrm{Cl}$ : $2.254-10.668, p<0.001)$ were identified as independent prognostic factors in patients with VEGFR-3 positive tumors. (B) For recurrence-free survival, MLVD analyzed by interquartile range $(95 \% \mathrm{Cl}$ : $1.942-8.389, p<0.001)$ revealed independent prognostic significance. (C) In 77 patients with VEGFR-3 positive tumors, higher level of MLVD was closely correlated with negative HBV infection, lymph node metastasis and tumor recurrence $(U$-test, $p=0.002,0.003$ and $<0.001$, respectively). Box-scatter plot graphics display a statistical summary of the median, quartiles and ranges of MLVD. 


\section{Discussion}

Intrahepatic cholangiocarcinoma is considered to be a highly aggressive carcinoma with poor prognosis due to early invasion, lymph node metastasis, rapid progression of tumor and frequent recurrence after surgical operation $[2,5,15]$. The lack of appropriate medical approaches calls for identifying effective prognostic biomarkers which influence the progression and metastasis of iCCA. VEGFR-3, a tyrosine kinase receptor for VEGF-C/ VEGF-D, is considered to be a major driver of embryonic vascular development. In tumor entities, over-expression of VEGFR-3 was reported to stimulate lymph node metastasis and promote proliferation, mobility and invasion of cancer cells as well $[16,17]$. However, expression of VEGFR-3 and its clinicopathological and prognostic significance in iCCA is far from known.

In this study, 77 of 106 patients had VEGFR-3 positive expression in tumor cells, providing a positive rate of $72.6 \%$. VEGFR-3 positive expression was significantly associated with tumor number, lymph node metastasis and tumor recurrence. In addition, patients with positive VEGFR-3 tumors demonstrated significantly worse overall and recurrence-free survival and VEGFR-3 expression was established as an independent predictor of survival in patients with iCCA after resection. However, it is noteworthy that neither intensity nor percentage of VEGFR-3 staining was prognostic factors for survivals, which provided proof that VEGFR-3 expression assessed by combination of intensity and percentage was a superior prognostic factor. The results are partly consistent with previously published studies in prostate [18] and non-small-cell lung cancer $[13,19]$. It is therefore important to determine the significant role of VEGFR-3 positive expression in iCCA.

However, it is important to note that VEGFR-3 expression showed no correlation with clinical parameters involved in local tumor growth including tumor size, histological differentiation, vascular invasion and tumor distribution. The different influence of VEGFR-3 mainly arises from the unique biological characteristics of iCCA. iCCA, a solid tumor lack of blood supply, is characterized by lymph node metastasis $[1,2,5]$. VEGFR-3 has been confirmed to promote lymph node metastasis in experimental models [16], while clinical parameters including tumor size, bilobar distribution and vascular invasion are more likely to be influenced by tumor-associated angiogenesis in hepatobiliary tumors [20, 21, 22]. It may thus explain that in iCCA entities, VEGFR-3-induced lymph node metastasis facilitates tumor recurrence and poor prognosis instead of local tumor growth affected by angiogenesis.

In addition, our study further found that higher proportion of micro-lymphatic vessel density (MLVD) was significantly associated with lymph node metastasis, tumor recurrence and VEGFR-3 positive expression in tumors. It revealed that VEGFR-3 expression in tumors may facilitate lymph node metastasis through tumor-associated lymphangiogenesis. As known, the VEGFR-3-VEGFC/VEGFD axis is the most studied lymphangiogenic signaling pathway. Many studies of tumor xenograft models in mice have shown that targeting this pathway by use of a soluble form of VEGFR3 or specific monoclonal antibodies restricts lymphangiogenesis and lymph node metastasis [10, 11, 23, 24]. Moreover, increasing evidence has shown that pre-existing lymphatics in cancer undergo lymphangiogenesis to favor entry of tumor cells into the lymphatic vasculature just before lymph node metastasis has occurred [25]. Studies of human malignant breast cancer [12, 26, 27] and colorectal cancer [28] indicated that cancers with high lymphatic vessel density invaded lymphatic vessels significantly more often and correlated with reduced overall survival. Since our study discovered that patients with VEGFR-3 positive tumors had significantly higher tumor-associated lymphangiogenesis and VEGFR-3 expression was closely related to lymph node metastasis, it is first deduced that positive expression of VEGFR-3 in tumors favors lymph node metastasis and worse outcome by promoting tumor-associated lymphangiogenesis in hepatic resected patients with iCCA.

It is interesting to find that HBV infection was identified as an independent positive prognostic factor for recurrence-free survival and was closely related to lower tumor-associated lymphangiogenesis. This finding is line with several results that consider HBV infection as a favorable factor in iCCA. Zhang et al. [29] reported that iCCA patients with HBV infection had a significantly better prognosis than patients without $\mathrm{HBV}$ infection and suggested that activated immune responses by HBV infection may enhance anti-tumor activity against iCCA. Another study conducted a mathematical model for predicting survival and concluded HBV infection as a positive factor of iCCA patients after hepatectomy [30]. On the other side, Tao et al. [31] described that 1-, 3-, and 5-year survival rates of HBsAg-positive iCCA patients were significantly lower than HBV-negative iCCA patients, which was contrary to our results. The different results may be attributed to limited number of cohort and patients enrolled from different stages of tumor upon diagnosis. In our cohort, patients with HBV infection revealed early detection of unexpected tumors because of regular follow-up of HBV infection, 
whereas majority of iCCA patients without HBV infection represented with advanced stage of tumors due to lack of early symptoms and detection. Thus, our results of HBV infection need to be further confirmed by multicenter large-scale studies and stratified by different stages of tumor since detection of tumors.

Recently, various inhibitors that target the VEGFR-3-VEGFC/VEGFD axis have been developed that might be useful in clinic. The neutralizing antibodies that target VEGFR-3 have progressed to Phase I clinical trials in patients with advanced solid tumors in 2013 [32]. Several small molecule protein kinase inhibitors (PKIs) such as sorafenib [33], pazopanib [34] and sunitinib [35, 36] have been developed that target various kinases, including VEGFR-3. However, none of these has been studied in patients with iCCA. Based on our results, iCCA is a highly malignant tumor with limited therapeutic approaches except hepatic resection. Since VEGFR-3 positive expression in tumors could serve as a negative prognostic marker for iCCA, hepatic resected patients with VEGFR-3 positive tumors are suggested to be then offered an anti-VEGFR-3 strategy to reduce the incidence of recurrence. In addition, iCCA might seed lymph node metastasis from an early stage or a small size. As VEGFR-3 expression was found to facilitate lymph node metastasis through lymphangiogenesis in our study, pre-operative anti-VEGFR-3 drugs could also be used in theory for treatment of patients with advanced cancers. More animal-based studies and clinical trials are required to identify the efficacy of anti-VEGFR-3 strategy for iCCA in future.

This study provides a great deal of information about the pivotal role of VEGFR-3 positive tumors in hepatic resected patients with iCCA. However, there are a few underlying limitations that remain to be confirmed. Firstly, the limited number of patients needs additional multicenter large-size studies to confirm the results. Secondly, all patients were based on Chinese population, so the results need to be further validated by patients from other regions. In addition, patients enrolled in this study were locally restricted malignancies in the liver. More detailed results should be analyzed on patients present with advanced or metastatic tumors. Despite the above limitations, our study is the first to represent VEGFR-3 positive tumors is related to poor prognosis of iCCA through tumor-associated lymphangiogenesis.

\section{Conclusions}

Expression of VEGFR-3 positive tumors represents an independent prognostic factor for both overall and recurrence-free survival in surgical resected patients with iCCA. Also, VEGFR-3 positive expression favors lymph node metastasis and tumor recurrence through tumor-associated lymphangiogenesis. Due to limited number of patients and retrospective nature, our study calls for validation by multicenter studies.

\section{Abbreviations}

VEGFR-3: Vascular endothelial growth factor receptor-3; iCCA: Intrahepatic cholangiocarcinoma; MLVD: Micro-lymphatic vessel density; HBV: Hepatitis B virus; AFP: Alpha-fetoprotein; CA19-9: Carbohydrate antigen 19-9.

\section{Supplementary Material}

Supplementary figures and tables. http://www.ijbs.com/v14p1333s1.pdf

\section{Acknowledgements}

We express appreciation for language help and writing assistance from Xiao-ni Kong and manuscript revising from Qiang Xia.

\section{Financial support}

This work was supported by the National Natural Science Foundation of China (81472234, 81670598) to Qiang Xia, and the Shanghai Health Bureau Key Joint Efforts Foundation (2013ZYJB001) to Qiang Xia.

\section{Authors' contributions}

Meng Sha and Seogsong Jeong conducted the present study. Meng Sha drafted the manuscript. Meng Sha, Seogsong Jeong, Xiao-song Chen, Ying Tong, Jie Cao, Han-yong Sun, Lei Xia, Ning Xu, Xin Wang, Long-zhi Han and Zhi-feng Xi collected clinical data. Meng Sha and Xiao-song Chen performed the statistical analyses. Meng Sha, Seogsong Jeong, Xiao-song Chen, Ying Tong, Jie Cao, Han-yong Sun Lei Xia, Ning Xu, Xin Wang, Long-zhi Han, Zhi-feng $\mathrm{Xi}$ and Jian-jun Zhang participated in the study design and concept. Xiao-ni Kong and Qiang Xia revised the manuscript critically for intellectual content. All authors read and approved the final manuscript.

\section{Competing Interests}

The authors have declared that no competing interest exists.

\section{References}

1. Bridgewater J, Galle PR, Khan SA, Llovet JM, Park JW, Patel T, et al. Guidelines for the diagnosis and management of intrahepatic cholangiocarcinoma. J Hepatol. 2014; 60: 1268-89.

2. Razumilava N, Gores GJ. Cholangiocarcinoma. Lancet. 2014; 383: 2168-79. 
3. Mavros MN, Economopoulos KP, Alexiou VG, Pawlik TM. Treatment and Prognosis for Patients With Intrahepatic Cholangiocarcinoma: Systematic Review and Meta-analysis. JAMA Surg. 2014; 149: 565-74.

4. Uenishi T, Yamamoto T, Takemura S, Kubo S. Surgical treatment for intrahepatic cholangiocarcinoma. Clin J Gastroenterol. 2014; 7: 87-93.

5. Rizvi S, Gores GJ. Pathogenesis, diagnosis, and management of cholangiocarcinoma. Gastroenterology. 2013; 145: 1215-29.

6. Howell M, Valle JW. The role of adjuvant chemotherapy and radiotherapy for cholangiocarcinoma. Best Pract Res Clin Gastroenterol. 2015; 29: 333-43.

7. Secker GA, Harvey NL. VEGFR signaling during lymphatic vascular development: From progenitor cells to functional vessels. Dev Dyn. 2015; 244: 323-331.

8. Chen JC, Chang YW, Hong CC, Yu YH, Su JL. The role of the VEGF-C/VEGFRs axis in tumor progression and therapy. Int J Mol Sci. 2012; 14: 88-107.

9. Tammela T, Alitalo K. Lymphangiogenesis: Molecular mechanisms and future promise. Cell. 2010; 140: 460-476.

10. Tvorogov D, Anisimov A, Zheng W, Leppänen VM, Tammela T, Laurinavicius $\mathrm{S}$, et al. Effective suppression of vascular network formation by combination of antibodies blocking VEGFR ligand binding and receptor dimerization. Cancer Cell. 2010; 18: 630-640.

11. Pytowski B, Goldman J, Persaud K, Wu Y, Witte L, Hicklin DJ, et al. Complete and specific inhibition of adult lymphatic regeneration by a novel VEGFR-3 neutralizing antibody. J Natl Cancer Inst. 2005; 97: 14-21.

12. Raica M, Cimpean AM, Ceausu R, Ribatti D. Lymphatic microvessel density, VEGF-C, and VEGFR-3 expression in different molecular types of breast cancer. Anticancer Res. 2011; 31: 1757-64.

13. Li J, Yi H, Liu Z, Zhang H, Zhang D, Yue W, et al. Association between VEGFR-3 expression and lymph node metastasis in non-small-cell lung cancer. Exp Ther Med. 2015; 9: 389-394.

14. Yokoyama Y, Charnock-Jones DS, Licence D, Yanaihara A, Hastings JM, Holland CM, et al. Expression of vascular endothelial growth factor (VEGF)-D and its receptor, VEGF receptor 3, as a prognostic factor in endometrial carcinoma. Clin Cancer Res. 2003; 9: 1361-9.

15. Esnaola NF, Meyer JE, Karachristos A, Maranki JL, Camp ER, Denlinger CS. Evaluation and management of intrahepatic and extrahepatic cholangiocarcinoma. Cancer. 2016; 122: 1349-69.

16. He Y, Rajantie I, Pajusola K, Jeltsch M, Holopainen T, Yla-Herttuala S, et al. Vascular endothelial cell growth factor receptor 3-mediated activation of lymphatic endothelium is crucial for tumor cell entry and spread via lymphatic vessels. Cancer Res. 2005; 65: 4739-46.

17. Matsumoto M, Roufail S, Inder R, Caesar C, Karnezis T, Shayan R, et al. Signaling for lymphangiogenesis via VEGFR-3 is required for the early events of metastasis. Clin Exp Metastasis. 2013; 30: 819-32

18. Jennbacken K, Vallbo C, Wang W, Damber JE, et al. Expression of vascular endothelial growth factor C (VEGF-C) and VEGF receptor-3 in human prostate cancer is associated with regional lymph node metastasis. Prostate. 2005; 65: $110-6$

19. Donnem T, Al-Shibli K, Al-Saad S, Delghandi MP, Busund LT, Bremnes RM. VEGF-A and VEGFR-3 correlate with nodal status in operable non-small cell lung cancer: inverse correlation between expression in tumor and stromal cells. Lung Cancer. 2009; 63: 277-83.

20. Thelen A, Scholz A, Weichert W, Wiedenmann B, Neuhaus P, Gessner R, et al. Tumor-associated angiogenesis and lymphangiogenesis correlate with progression of intrahepatic cholangiocarcinoma. Am J Gastroenterol. 2010; 105: 1123-1132.

21. Murakami K, Kasajima A, Kawaqishi N, Ohuchi N, Sasano H. Microvessel density in hepatocellular carcinoma: Prognostic significance and review of the previous published work. Hepatol Res. 2015; 45: 1185-1194.

22. Ribatti D, Vacca A, Nico B, Sansonno D, Dammacco F. Angiogenesis and anti-angiogenesis in hepatocellular carcinoma. Cancer Treat Rev. 2006; 32: 437-44.

23. He Y, Kozaki K, Karpanen T, Koshikawa K, Yla-Herttuala S, Takahashi T, Alitalo K. Suppression of tumor lymphangiogenesis and lymph node metastasis by blocking vascular endothelial growth factor receptor 3 signaling. J Natl Cancer Inst. 2002; 94: 819-25.

24. Quagliata L, Klusmeier S, Cremers N, Pytowski B, Harvey A, Pettis RJ, Thiele $\mathrm{W}$, Sleeman JP. Inhibition of VEGFR-3 activation in tumor-draining lymph nodes suppresses the outgrowth of lymph node metastases in the MT-450 syngeneic rat breast cancer model. Clin Exp Metastasis. 2014; 31: 351-365.

25. Stacker SA, Williams SP, Karnezis T, Shayan R, Fox SB, Achen MG. Lymphangiogenesis and lymphatic vessel remodeling in cancer. Nat Rev Cancer. 2014; 14: 159-172

26. Gu Y, Qi X, Guo S. Lymphangiogenesis induced by VEGF-C and VEGF-D promotes metastasis and a poor outcome in breast carcinoma: a retrospective study of 61 cases. Clin Exp Metastasis. 2008; 25: 717-25.

27. Nakamura $Y 1$, Yasuoka H, Tsujimoto M, Imabun S, Nakahara M, Nakao K, et al. Lymph vessel density correlates with nodal status, VEGF-C expression, and prognosis in breast cancer. Breast Cancer Res Treat. 2005; 91: 125-32.

28. Matsumoto K, Nakayama Y, Inoue Y, Minagawa N, Katsuki T, Shibao K, Tsurudome Y, Hirata K, Nagata N, Itoh H. Lymphatic microvessel density is an independent prognostic factor in colorectal cancer. Dis Colon Rectum. 2007; 50: 308-14.
29. Zhang L1, Cai JQ Zhao JJ, Bi XY, Tan XG, Yan T, Li C, Zhao P. Impact of hepatitis $B$ virus infection on outcome following resection for intrahepatic cholangiocarcinoma. J Surg Oncol. 2010; 101: 233-8.

30. Jiang BG, Ge RL, Sun LL, Zong M, Wei GT, Zhang YJ. Clinical parameters predicting survival duration after hepatectomy for intrahepatic cholangiocarcinoma. Can J Gastroenterol. 2011; 25: 603-8.

31. Tao LY, He XD, Xiu DR. Hepatitis B virus is associated with the clinical features and survival rate of patients with intrahepatic cholangiocarcinoma. Clin Res Hepatol Gastroenterol. 2016; 40: 692-687.

32. Persaud K, Tille JC, Liu M, Zhu Z, Jimenez X, Pereira DS, et al. Involvement of the VEGF receptor 3 in tubular morphogenesis demonstrated with a human anti-human VEGFR-3 monoclonal antibody that antagonizes receptor activation by VEGF-C. J Cell Sci. 2004; 117: 2745-56.

33. Bruix I, Raoul JL, Sherman M, Mazzaferro V, Bolondi L, Craxi A, et al. Efficacy and safety of sorafenib in patients with advanced hepatocellular carcinoma: subanalyses of a phase III trial. J Hepatol. 2012; 57: 821-9.

34. Motzer RJ, Hutson TE, Cella D, Reeves J, Hawkins R, Guo J, et al. Pazopanib versus sunitinib in metastatic renal-cell carcinoma. N Engl J Med. 2013; 369: 722-31.

35. Thomas A, Rajan A, Berman A, Tomita Y, Brzezniak C, Lee MJ, et al. Sunitinib in patients with chemotherapy-refractory thymoma and thymic carcinoma: an open-label phase 2 trial. Lancet Oncol. 2015; 16: 177-86.

36. Ravaud A, de la Fouchardière C, Caron P, Doussau A, Do Cao C, Asselineau J, et al. A multicenter phase II study of sunitinib in patients with locally advanced or metastatic differentiated, anaplastic or medullary thyroid carcinomas: mature data from the THYSU study. Eur J Cancer. 2017; 76: 110-117. 